\title{
Robotic hand design with linear actuators based on Toronto development
}

\author{
Vargas Oscar ${ }^{1}$ \\ ORCID: 0000-0003-4036-7280 \\ oscar3vargas@gmail.com \\ Universidad Internacional del Ecuador. \\ Ingeniería Mecatrónica \\ Quito- Ecuador. \\ Toapanta Carlos ${ }^{3}$ \\ ORCID: 0000-0001-6156-5222 \\ cartoap@gmail.com \\ Universidad de las Fuerzas
}

\author{
Flor Omar ${ }^{2}$ \\ ORCID: 0000-0002-3455-5982 \\ omar.flor@udla.edu.ec \\ Universidad de las Américas \\ Facultad de Ingeniería y \\ Ciencias Aplicadas \\ Quito-Ecuador
}

Recibido (08/08/20), Aceptado (21/08/20)

\begin{abstract}
In this work, the design of a robotic hand with 7 degrees of freedom is presented that allows greater flexibility, achieving the usual actions performed by a normal hand. The work consists of a prototype designed with linear actuators and myoelectric sensor, following the mechanism of the University of Toronto for the management of functional phalanges. The design, construction description, components and recommendations for the elaboration of a flexible and useful robotic hand for amputee patients with a residual limb for the socket are presented.
\end{abstract}

Keywords: Robotic hand, Degree of freedom, Toronto's Mechanism, lineal actuator.

\section{Diseño de mano robótica con actuadores lineales basados en el desarrollo de Toronto}

Resumen: En este trabajo se presenta el diseño de una mano robótica de 7 grados de libertad que permite mayor flexibilidad, logrando las acciones habituales realizadas por una mano normal. El trabajo consta de un prototipo diseñado con actuares lineales y sensor mioeléctrico, siguiendo el mecanismo de la Universidad de Toronto para el manejo de falanges funcionales. Se presenta el diseño, descripción constructiva, componentes y recomendaciones para la elaboración de una mano robótica flexible y útil para pacientes amputados con miembro residual para el encaje.

Palabras Clave: Mano robótica, grado de libertad, Mecanismo de Toronto, actuador lineal. 


\section{I.INTRODUCCIÓN}

$3 \%$ of the amputations performed in the United States correspond to upper limbs, involving affectations that affect and limit people's vocational development [1]. Forearm amputations represent a significant number each year around the world. The cost of myoelectric control prosthesis is relatively high and proportional to the number of degrees of freedom [2]. Recent advances have achieved articulated finger configurations [3,4].

In this work we present a design adapted to linear actuators that drive a mechanism design based on the University of Toronto mechanism [5]. And it can be played back using the files found in reference [6]. The proposed prosthesis is presented in Figure 1.

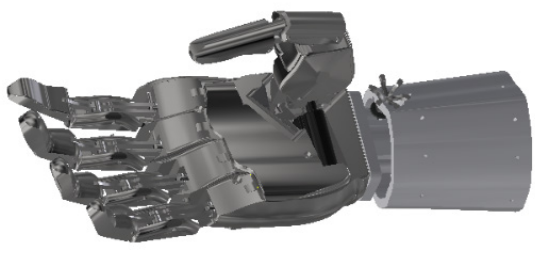

Fig.1. 7DOF hand prosthesis prototype

The Toronto development [5] has been considered because it allows a greater similarity to the human hand, offering three articulated phalanges, including the thumb.

For this design some functional aspects were considered given the experience of previous prototypes $[7,8,9]$. Given the limited space of the real human hand and in order not to use high-cost and energy-consuming devices, mid-range devices have been used and easily obtained in houses specialized in electronics.

One of the important considerations is an arrangement of the thumb that allows mobility in 2 directions of rotation, achieving abduction and adduction of the thumb. With this it is possible to improve the grip of pieces, placing this finger in a more natural position.

Most prostheses of this type have wide mobility configurations [10]. For this reason, it has been considered to work with a ball joint that allows rotation in 3 axes, as evidenced in works developed [11-14].

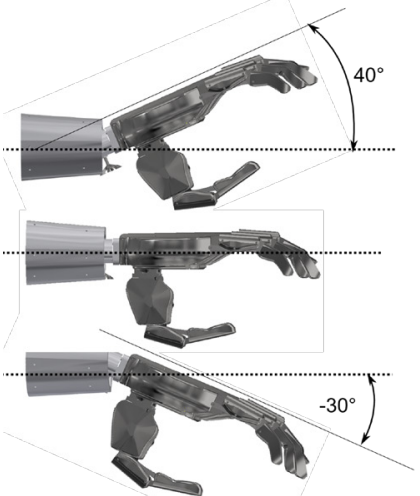

(a)

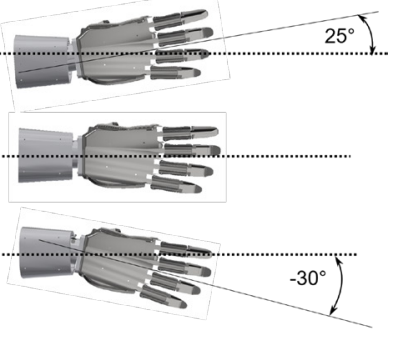

(b)

Fig. 2. Degrees of mobility of (a) wrist of the hand, (b) thumb. 


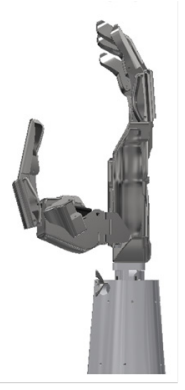

(a)

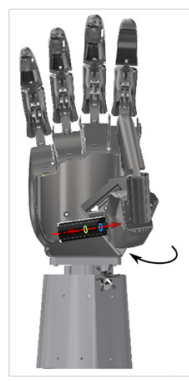

(b)

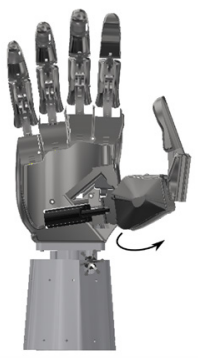

(c)

Fig. 3. (a) Lateral view, (b) abduction movement and (c) adduction movement of the thumb.

Figure 4 shows the breakdown of the parts that make up the prototype, it shows the mechanical and electronic elements that make the movement of the hand possible.

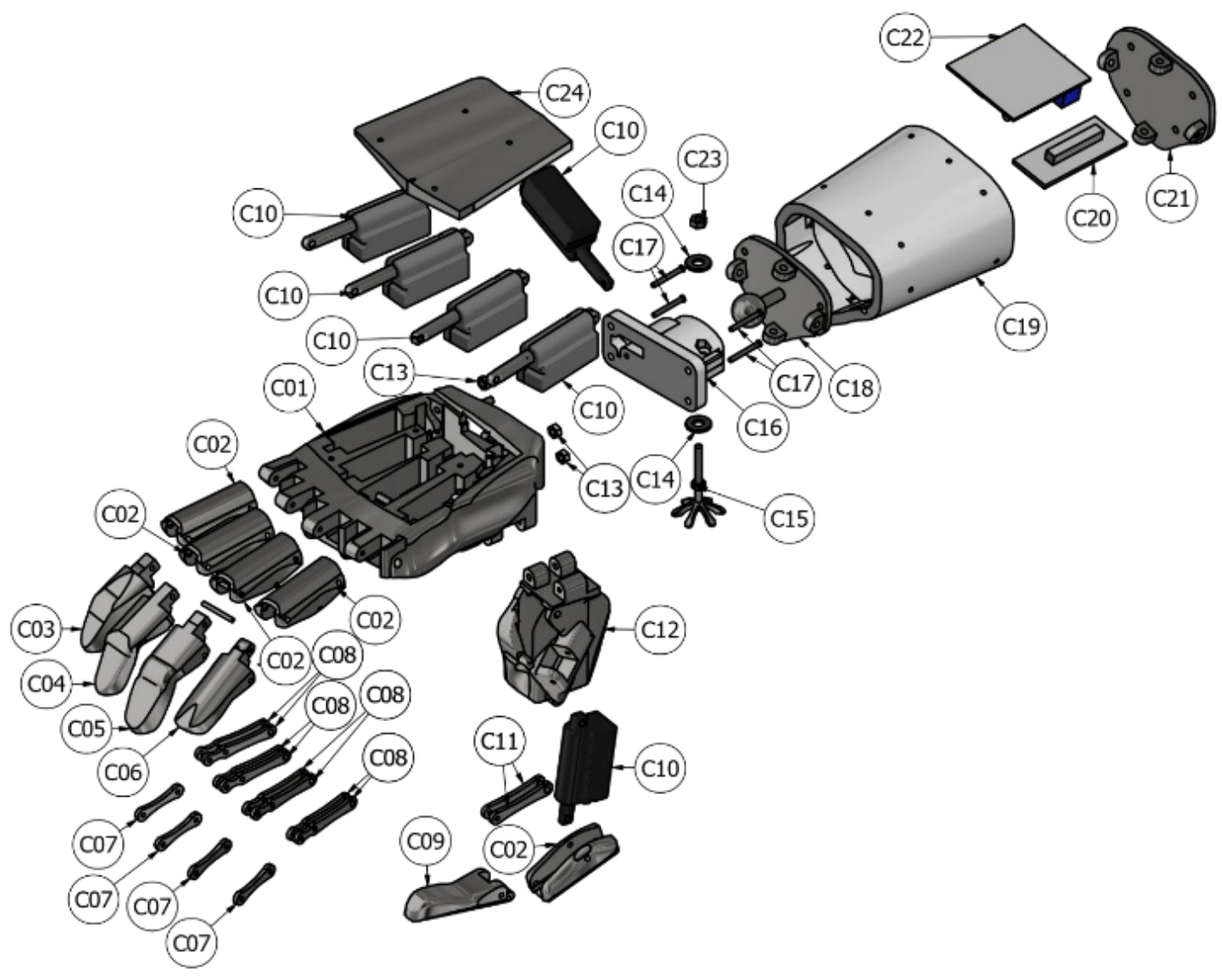

Fig. 4. Assembly diagram of the robotic hand.

\section{II.METHODS AND MATERIALS}

\section{A.Methods.}

The methods used to obtain the parts for the manufacture of the design can be obtained through the use of some existing technologies in machine shops, machining centers and cutting plotters. These are: (P1) 3D printing, (P2) stainless steel water jet cutting, (P3) stainless steel laser cutting, (P4) stainless steel welding, (P5) 
bolted joint, (P6) steel turning .

\section{B.Materials}

For the pieces to be obtained by P1, poly lactic acid (PLA) will be used, while for the pieces obtained manufactured by $\mathrm{P}_{2}$ and $\mathrm{P} 3$, stainless steel with a thickness of $2 \mathrm{~mm}$ will be used. The mobile ball joint that will be incorporated in the $\mathrm{C} 18$ component, machine steel is used and through the $\mathrm{P} 6$ process, it is machined generating the appropriate shape. The bolts for the joints shall be stainless steel.

\section{III.ASSEMBLY PROCESS}

Element C01 corresponds to the Palm. The C10 servomotors are inserted into the respective cavities being snap secured. The C02 Proximal Phalanges are secured to the Co1 body by means of E1 stainless steel shafts of $2 \mathrm{~mm}$ diameter and $18 \mathrm{~mm}$ long. The Medial Phalanx Littlefinger (C03), Annular (C04), Middle (Co5), Index (C06) are coupled to Co2 components using E1 stainless steel shafts. The C07 Medial Links join the Co3, C04, C05, C06 Medial Phalanges by means of E1, C07 stainless steel shafts also join the Co8 Proximal Phalanges with stainless steel shafts of $2 \mathrm{~mm}$ diameter, length $7 \mathrm{~mm}$ E2. The C08 Proximal Links are fixed to Co2 with E1 stainless steel shafts, in addition they are inserted into the $\mathrm{C} 10$ servo motor shafts with $2.6 \mathrm{~mm}$ diameter stainless steel shafts, $6 \mathrm{~mm}$ E3 length. The Thumb Medial Phalanx Co9 to Co2 is attached and the latter to the C12 Thumb Base with E1 shafts, $\mathrm{C} 12$ to $\mathrm{Co} 1$ is fixed with a $2 \mathrm{~mm}$ diameter stainless steel shaft, $24 \mathrm{~mm}$ E4 length. The Medial Thumb Link $\mathrm{C} 11$ is attached to $\mathrm{Co} 9$ and $\mathrm{Co} 2$ with $\mathrm{E} 1$ axes. C10 is inserted into the cavity of $\mathrm{C} 12$ and fixed with a 3mm diameter stainless steel shaft, 23mm length E5. Attach the C16 Ball Coupling to Co1 with the C17 Bolts and $\mathrm{C} 13$ Nuts. Attach the C18 Ball Joint to the C19 Forearm Coupling with 4 M3 bolts by $15 \mathrm{~mm}$ length E6. The Control Board C22 and the Arduino C20 controller are inserted into the cavity of C19 and closed with the C21 Cover Forearm Coupling, this is fastened with 4 E6 bolts. Insert $\mathrm{C} 18$ to $\mathrm{C} 16$ and tighten with the M3 bolt for 20mm length E7 with the M3 C23 nut. For this bolt the flanges (C14) are used for the M3 bolt. Finally, the Palm Cap C24 is placed on C01, it is secured with $4 \mathrm{M} 6$ bolts by 30mm in length, E8 with their respective nuts.

\section{A.Moving joints}

The prototype has fixed parts and moving parts. The moving part corresponds to the Toronto mechanism to transmit the movement to the fingers, stainless steel shafts are fixed between the phalanges and the palm of the hand $\mathrm{CO} 1$.

\section{B.Piece joints}

The C01, constitutes the support of all the mobile mechanisms and internal actuators that propel the fingers. The clearance presented by the internal components conveniently ensures the linear servomotors, while the C22 and $\mathrm{C} 20$ electronic control devices are supported in the forearm portion C19. Furthermore, in C19 the socket is coupled to the residual limb (part of the forearm) of the user, whose part is designed in a particular way for each user.

\section{C.Electronic equipment}

In previous prototypes, use had been made of MyoWare myoelectric sensors which take the user's myoelectric signal, amplify, filter and rectify said signal and in this way the sensor output signal can be read directly through the analog input ports of the a microcontroller, they are also affordable. The drawback that was had in the tests that was carried out on the prototypes was that the user with time of use begins to present an allergy to the use of the electrode, in addition, these electrodes need to be replaced every time the prosthesis is going to be used, since They lose their rubber adherence to the user's skin. For these drawbacks, a sensor with dry electrodes such as the Myo bracelet is sought. 


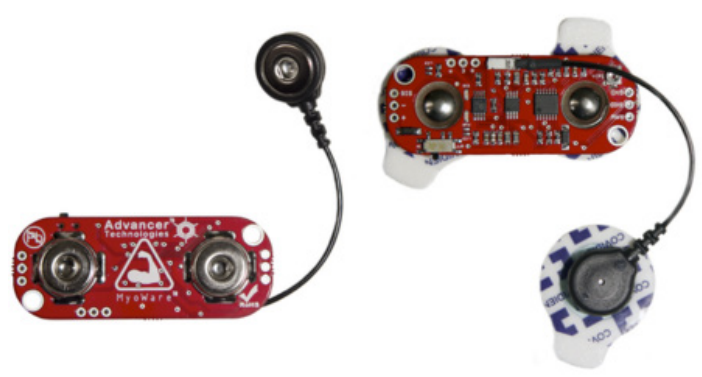

Fig. 5. MyoWare Sensor

To control the prosthesis, it is done using free software and hardware, such as Arduino, thus reducing licensing costs and there are also libraries for the realization of the interface with the myoelectric bracelet "Myo Armband" with which the signals from the muscles of the prosthesis wearer. The data acquisition bracelet is in charge of carrying out the amplification and filtering stages of the myoelectric signals obtained from the user, as well as their analysis and processing through "machine learning"; thus the interface between the controller and the bracelet serves as a means of communication for sending commands for five types of movements.

The library that allows communication between the bracelet and the controller is MyoBridge, also the Arduino needs a bluetooth module HM-10 or HM-11 that has the CC2541 chip to which a "firmware" must be loaded, with an Arduino, called CCLoader, once the entire configuration process has been carried out in which it is necessary to make extra connections in the bluetooth module, all this is explained in [15] it is necessary to perform all the steps so that the interface on the bracelet and the Arduino controller are set.

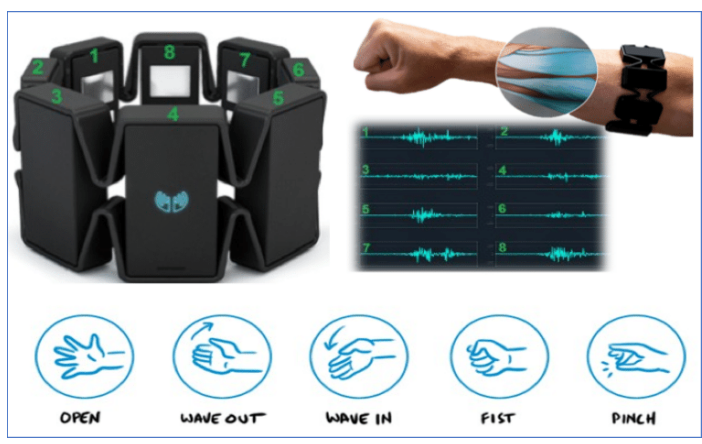

Fig. 6. Myo bracelet with the movements it recognizes in the user.

The Myo bracelet is configured in its Myo Connect application which can be downloaded from its page [16], through this application the Myo bracelet is trained to recognize the five patterns that the user is going to make, once they have been recognized the patterns, the bracelet communicates them through the HM-11 bluetooth module to the Arduino controller in which this data is received through RS232 serial communication and processed for the control of six Actuonix PQ12-R linear servo motors, which are It controls with a PWM signal where the bandwidth for the opening or closing of the servomotors is varied, which in turn move the fingers of the prosthesis.

\section{IV.RESULTS}

The proposed design presents constructive feasibility, low cost of both electronic and structural components, due to the use of simple technologies to find in any region or country.

The mobility developed by the 7 degrees of freedom robotic hand has improvements in movement dexterity, improving 5 degrees of freedom prototypes. Given the movements of the thumb, small pieces can be manipulated, especially to hold them.

Fine motor skills and the fine movement of the fingertips allow the holding of fine items such as sheets of paper in conjunction with the thumb. 
An improvement in battery life is offered given the use of low consumption devices and the Myo bracelet which is independent from the rest of the electronic devices in the bracelet, this also helps to reduce the workload of the controller and avoid failures due to Processing overhead on both the Myo armband and the Arduino controller.

Separating the processing of myoelectric signals with the control of the movement of the prosthesis is optimal due to the large amount of data and functions that are carried out in pattern recognition; In addition to generating the PWM signal for the control of servo motors, it interferes with the myoelectric signals coming from the user's forearm.

The recognition of movement patterns of the user through its myoelectric signals becomes almost impossible to do when trying to do it using traditional programming logics because there is a great variability of said signals due to various factors, which is why it is necessary to use Non-traditional and even experimental methods such as fuzzy logic and "machine learning" for the processing and recognition of patterns to be effective, as the Myo bracelet does, this in turn requires powerful processors which is difficult to achieve in microcontrollers that also need to perform additional functions, it is important to separate this recognition process from the rest of the functions.

The mass of the prosthesis does not exceed eight hundred grams, being similar to the mass of the lost limb of the user, this factor is important because the use of the device must be continuous and the user must be given the greatest possible comfort.

The movement of the wrist is necessary because the prosthesis must grasp objects in different positions for which it is important that the prosthesis adapts to the natural grip position of a person, otherwise the user must perform movements to accommodate and make the grips in different positions, these with continuous use tire the user causing him to stop using the prosthesis since it is not helpful.

\section{V.CONCLUSIONS}

This design proposes a solution adapted to the technology of manufacturing by deposition of molten plastic material, with few components in steel, which must be manufactured in specialized workshops. These features allow this project to be replicated and used by people who lack their hand and who have a residual limb for fitting, as detailed in the figures.

The presented robotic hand presents a sufficient and necessary flexibility for multiple operations of the hand, in addition its configuration improves the grip of small objects providing the performance of fine motor skills by the user.

The elaboration of the parts has been a rigorous process of computer-aided design, limiting the design to the linear actuators used and explained in the development. In this work, the interior space has been optimized, allowing that in subsequent works to design, without inconvenience, covers that have dimensions of a real hand.

\section{ACKNOWLEDGMENT}

Thanks to the University of the Americas and especially to the industrial engineering laboratory for their support in the manufacturing process and supply of materials.

\section{REFERENCES}

[1]W. Diane, J. Braza and M. Yacub, Essentials of Physical Medicine and Rehabilitation, 4th ed. Philadelphia: Walter R. Frontera and Julie K. Silver and Thomas D. Rizzo, 2020, pp. 651 - 657.

[2]A. Heerschop, C. Van Der Sluis, E. Otten, \& R.M. Bongers, Looking beyond proportional control: The relevance of mode switching in learning to operate multi-articulating myoelectric upper-limb prostheses, . Biomedical Signal Processing and Control, 2020, doi:10.1016/j.bspc.2019.101647

[3]L. Heisnam, B. Suthar, 20 DOF robotic hand for tele-operation: - Design, simulation, control and accuracy test with leap motion. 2016 International Conference on Robotics and Automation for Humanitarian Applications (RAHA), 2016, doi:10.1109/raha.2016.7931886

[4]Y. Mishima, R. Ozawa, Design of a robotic finger using series gear chain mechanisms. 2014 IEEE/RSJ International Conference on Intelligent Robots and Systems, 2014, doi:10.1109/iros.2014.6942961.

[5]N. Dechev, W. Cleghorn, S. Naumann, Multi-segmented finger design of an experimental prosthetic hand, 
Proceedings of the Sixth National Applied Mechanisms \& Robotics Conference, december 1999.

[6]O. Flor, "Building a mobile robot," Education for the future. Accessed on: December 29, 2019. [Online] Available: https://omarflor2014.wixsite.com/misitio.

[7]Vargas, O., Flor,O., Suarez, F., Design of a robotic prototype of the hand and right forearm for prostheses, Universidad, Ciencia y Tecnología, 2019.

[8]O. Vargas, O. Flor, F. Suarez, C. Chimbo, Construction and functional tests of a robotic prototype for human prostheses, Revista espirales, 2020.

[9]P. PonPriya, E. Priya, Design and control of prosthetic hand using myoelectric signal. International Conference on Computing and Communications Technologies (ICCCT), 2017, doi:10.1109/iccct2.2017.7972314

[10]N. Bajaj, A. Spiers, A. Dollar, State of the Art in Artificial Wrists: A Review of Prosthetic and Robotic Wrist Design. IEEE Transactions on Robotics, 2019, doi:10.1109/tro.2018.2865890

[11]R. Mahmoud, A. Ueno, and S. Tatsumi, "Dexterous mechanism design for an anthropomorphic artificial hand: Osaka City University Hand I," in Proc. 10th IEEE-RAS Int. Conf. Humanoid Robot. Humanoids, 2010, pp. 180-185.

[12]T. Inada, T. Tsujimori, S. Kitamura, and R. Taniuchi, "Robot," U.S. Patent 7622 001, 2009.

[13]N. Torii, K. Mizuno, and H. Iwasaki, "Wrist assembly for an industrial robot," U.S. Patent 4 972 735, 1990. [96]T. Hezel, I. Leiensetter, F. Herre, B. Maxh.

[14]K. A. Wyrobek, E. H. Berger, H. F. M. Van Der Loos, and J. K. Salisbury, "Towards a personal robotics development platform: Rationale and design of an intrinsically safe personal robot," in Proc. IEEE Int. Conf. Robot. Automat., 2008, pp. 2165-2170.

[15]Myo Armband using a HM-10/HM-1 1, january 29th, 2020. Available online: https://blog.raquenaengineering.com/arduino-and-the-myo-armband/

[16]Myo Connect, SDK and firmware downloads, january 29th, 2020. Available online: https://support.getmyo. com/hc/en-us/articles/360018409792

\section{CURRICULUM SUMMARY}

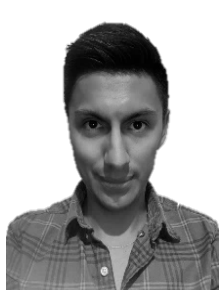

Oscar Vargas, Mechatronic Engineer from the International University of Ecuador, dedicated to the development of upper limb prostheses, designer and implementer of electrical and electronic systems for the oil industry at iNouxx Company in Quito - Ecuador.

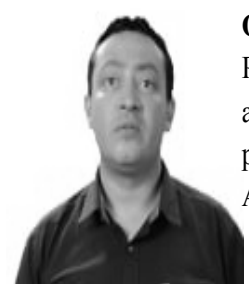

Omar Flor, Mechanical Engineer from the Army Polytechnic School, Master in Automation, Robotics and Telematics at the University of Seville-Spain, professor and researcher at the University of the Americas in Quito-Ecuador.

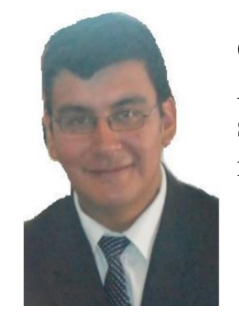

Carlos Toapanta, Mechanical Engineer from the Army Polytechnic School, experience in design, supervision, and quality assurance and control in the manufacturing and oil industry 